\title{
Editorial: Thermal and Non-Thermal Plasmas at Atmospheric Pressure
}

\author{
Antonio D'Angola ${ }^{1,2 *}$, Gianpiero Colonna ${ }^{2}$ and Elena Kustova ${ }^{3}$ \\ ${ }^{1}$ Scuola di Ingegneria SI, Università della Basilicata, Potenza, Italy, ${ }^{2}$ Institute for Plasma Science and Technology (ISTP), CNR, \\ Bari, Italy, ${ }^{3}$ Fluid Mechanics Department, Saint Petersburg State University, Saint Petersburg, Russia
}

Keywords: kinetic equations, ionized gas mixtures, plasma discharges, laser welding, plasma properties, plasma cancer treatment

\section{Editorial on the Research Topic}

\section{Thermal and Non-Thermal Plasmas at Atmospheric Pressure}

One of the typical classifications of plasma systems identifies two major categories: thermal and nonthermal plasmas, in which the temperatures of the different plasma species are not the same. Thermal plasma technology has evolved over the past decades due to the increasing attention in fields such as aerospace, microelectronics, automotive, material treatment and processing, melting and welding of metals, plasma chemical synthesis, and vapor deposition, plasma and arc spraying, and waste destruction [1]. Typical atmospheric plasma devices are realized by means of arcs or radio frequency (RF) inductively coupled plasma discharges [2], where the main phenomena involved are Joule heating and thermal ionization. Modeling these phenomena requires the knowledge of thermodynamic properties and transport coefficients of plasmas [3-8], which are of relevant importance, not only in equilibrium conditions. Numerical codes reliably evaluating these data can assist the designing and optimizing the phases of plasma-based devices. Over the past decades, several numerical approaches have been developed to investigate plasma behaviors and commercial multi-purpose codes are often used due to the complexity of systems. These numerical codes are based on the fluid dynamics approach, describing realistic discharge geometries. The main drawbacks of thermal plasmas are represented by low excitation selectivity and very high gas temperatures; serious quenching requirements and electrode problems result in limited energy efficiency and applicability of thermal plasma sources. For these reasons, non-thermal plasmas such as low-pressure glow and RF, microwave discharges, dielectric barrier discharges, and laser-produced plasmas have been used due to their high selectivity in plasma chemical reactions, operating effectively at low temperatures and without quenching. More recently, non-thermal atmospheric pressure plasmas have been studied for a variety of industrial [9] and medical applications [10] such as sterilization, ozone production for water purification, pollution control applications, car exhaust emission control, volatile organic compounds removal, and polymer surface treatment in order to improve properties such as wettability, printability, and adhesion.

The aim of this research topic, which contains four original contributions, is to present some innovative applications, enlarging the perspectives on thermal and non-thermal plasmas at atmospheric pressure, giving a guide on critical topics for the progress on plasma sources, diagnostic and modeling, investigating physical and chemical behavior of discharges both experimentally, theoretically and numerically and focusing attention on the industrial and medical applications. 
Rigorous theoretical models represent the basis for applied studies [11, 12]. Rydalevskaya and Voroshilova propose a new form of model kinetic equations for monoatomic gas mixtures taking into account multiple ionization. The main idea is to express the equilibrium distribution function in terms of the number of the corresponding chemical element in the periodic system and the electric charge of the particle. From this can be derived the closed set of conservation equations for the momentum, energy, number, and density of nuclei of different species, and the number and density of electrons, both bound and free. The closure of governing equations is conducted using the generalized Chapman-Enskog method. The proposed model is applicable for both local equilibrium and weakly non-equilibrium flows and provides an efficient tool for the evaluation of equilibrium multiply ionized mixture composition, adiabatic index, and sound velocity.

Recently, atmospheric pressure plasma is suggested as an alternative method for cancer treatment [13]. Its action deviates from the conventional chemotherapy drugs, by generating various active species such as charged particles, UV photons, ROS, and NOX which can eradicate cancer cells. Kim et al. describe the suppression of breast cancer cell migration by using a cold atmospheric pressure helium plasma consisting of a single copper pin electrode powered by an alternating current (AC) supply at $50 \mathrm{kHz}$ with a peak-to-peak voltage of $2.85 \mathrm{kV}$. Authors show experimentally that atmospheric pressure plasma treatment on an invasive breast cancer cell line alters these cells' morphology and further suppresses migratory activity reducing the invasive nature of cancer cells and in particular cancer metastasis.

Atmospheric or high-pressure plasma devices, mainly characterized by glow discharge, inductively coupled plasma discharges, or microwave-induced plasma, have become widely used in various fields, such as for cutting, spraying, welding, evaporation of a material, etching, or for the deposition of thin layers [14]. Maharaj et al. describe the dynamic behavior of a low current atmospheric and high-pressure arc plasma discharge by

\section{REFERENCES}

1. Boulos MI, Fauchais PL, Pfender E. Handbook of Thermal Plasmas. Springer International Publishing (2016). p. 1500.

2. Bernardi D, Colombo V, Coppa GGM, D'Angola A. Simulation of the Ignition Transient in RF Inductively-Coupled Plasma Torches. Eur Phys J D - At Mol Opt Plasma Phys (2001) 14:337-48. doi:10.1007/S100530170201

3. Colonna G, D’Angola A, Laricchiuta A, Bruno D, Capitelli M. Analytical Expressions of Thermodynamic and Transport Properties of the Martian Atmosphere in a Wide Temperature and Pressure Range. Plasma Chem Plasma Process (2013) 33:401-31. doi:10.1007/s11090-012-9418-4

4. Colonna G, D’Angola A, Pietanza LD, Capitelli M, Pirani F, Stevanato E, et al. Thermodynamic and Transport Properties of Plasmas Including Silicon-Based Compounds. Plasma Sourc Sci. Technol. (2018) 27:015007. doi:10.1088/13616595/aa9f9b

5. D’Angola A, Colonna G, Bonomo A, Bruno D, Laricchiuta A, Capitelli M. A Phenomenological Approach for the Transport Properties of Air Plasmas. Eur Phys J D (2012) 66:205. doi:10.1140/epjd/e2012-30147-8

6. D'Angola A, Colonna G, Gorse C, Capitelli M. Thermodynamic Properties of High Temperature Air in Local Thermodynamic Equilibrium: II Accurate comparing experimental results with those obtained with a Computational Fluid Dynamic modeling coupled with Maxwell's equations solver. The discharge electric potential has been calculated by considering the electrical characterization of the electrodes where the plasma increasingly deviates from equilibrium conditions. These devices can be used as plasma sources for wastewater treatment producing radicals and reactive species, which have been demonstrated to rapidly and efficiently degrade many organic compounds, especially in the plasma-liquid interface.

In recent decades, one of the most important developments of laser material processing in the field of advanced production methodologies regards joining processes [15]. Coviello et al. describe the influence of the plasma properties on the keyhole geometry in laser beam welding for a deep-penetration joining processes in which high incident laser beam power densities at the workpiece surface produce inside and above the keyhole cavity a mixture of ionized metal vapors (keyhole plasma). Plasma generation implicates absorption, scattering, and refraction of laser beam rays. The authors developed a numerical model to calculate the keyhole shape taking into account the effect of plasma properties during multiple reflections inside the cavity through an iterative ray-tracing technique.

These papers have shown a variety of applications of highpressure plasmas. In spite of the simplicity of the devices, the underlining physical-chemical phenomena are driven by the complex interplay between chemical reactions, field geometry, plasma-surface interaction, and radiation transport. In the next future, large efforts, from both theoretical and experimental research activities, will be necessary to shed light on the atmospheric and high-pressure plasma phenomena.

\section{AUTHOR CONTRIBUTIONS}

All authors listed have made a substantial, direct, and intellectual contribution to the work and approved it for publication.

Analytical Expression for Electron Molar Fractions. Eur Phys J D (2011) 65: 453-7. doi:10.1140/epjd/e2011-20424-5

7. D’Angola A, Colonna G, Gorse C, Capitelli M. Thermodynamic and Transport Properties in Equilibrium Air Plasmas in a Wide Pressure and Temperature Range. Eur Phys J D (2008) 46:129-50. doi:10.1140/epjd/ e2007-00305-4

8. Capitelli M, Colonna G, Gorse C, D'Angola A. Transport Properties of High Temperature Air in Local Thermodynamic Equilibrium. Eur Phys J D (2000) 11:279-89. doi:10.1007/s100530070094

9. Roth JR. Industrial Plasma Engineering: Vol. 2, Application to Non thermal Plasma Processing. Bristol, PA: IOP (2001). 660.

10. Fridman G, Shereshevsky A, Jost MM, Brooks AD, Fridman A, Gutsol A, et al. Floating Electrode Dielectric Barrier Discharge Plasma in Air Promoting Apoptotic Behavior in Melanoma Skin Cancer Cell Lines. Plasma Chem Plasma Process (2007) 27(2):163-76. doi:10.1007/s11090-007-9048-4

11. Loureiro J, Amorim J. Kinetics and Spectroscopy of Low Temperature Plasmas. Switzerland: Springer (2016). doi:10.1007/978-3-319-09253-9Kinetics and Spectroscopy of Low Temperature Plasmas

12. Istomin VA, Kustova EV. Transport Coefficients and Heat Fluxes in Nonequilibrium High-Temperature Flows with Electronic Excitation. Phys Plasmas (2017) 24:022109. doi:10.1063/1.4975315 
13. Gweon B, Kim M, Bee Kim D, Kim D, Kim H, Jung H, et al. Differential Responses of Human Liver Cancer and normal Cells to Atmospheric Pressure Plasma. Appl Phys Lett (2011) 99(6):063701. doi:10.1063/1.3622631

14. Bogaerts A, Neyts E, Gijbels R, van der Mullen J. Gas Discharge Plasmas and Their Applications. Spectrochim Acta B: At Spectrosc (2002) 57:609-58. doi:10. 1016/s0584-8547(01)00406-2

15. Svenungsson J, Choquet I, Kaplan AFH. Laser Welding Process - A Review of Keyhole Welding Modelling. Phys Proced (2015) 78:182-91. doi:10.1016/j. phpro.2015.11.042

Conflict of Interest: The authors declare that the research was conducted in the absence of any commercial or financial relationships that could be construed as a potential conflict of interest.
Publisher's Note: All claims expressed in this article are solely those of the authors and do not necessarily represent those of their affiliated organizations, or those of the publisher, the editors and the reviewers. Any product that may be evaluated in this article, or claim that may be made by its manufacturer, is not guaranteed or endorsed by the publisher.

Copyright $\odot 2022$ D'Angola, Colonna and Kustova. This is an open-access article distributed under the terms of the Creative Commons Attribution License (CC BY).

The use, distribution or reproduction in other forums is permitted, provided the original author(s) and the copyright owner(s) are credited and that the original publication in this journal is cited, in accordance with accepted academic practice. No use, distribution or reproduction is permitted which does not comply with these terms. 